\title{
Expression of P-EGFR and P-Akt protein in esophageal squamous cell carcinoma and its prognosis
}

\author{
ZHENG-ZHENG SHAN $^{1 *}$, PEI-NAN CHEN ${ }^{2 *}$, FENG WANG $^{1}$, JUN WANG $^{1}$ and QING-XIA FAN ${ }^{1}$ \\ ${ }^{1}$ Department of Oncology, The First Affiliated Hospital of Zhengzhou University; ${ }^{2}$ Department of Oncology, \\ The Second Affiliated Hospital of Zhengzhou University, Zhengzhou, Henan 450052, P.R. China
}

Received June 24, 2016; Accepted March 8, 2017

DOI: $10.3892 / 01.2017 .6526$

\begin{abstract}
The phosphorylated epidermal growth factor receptor (P-EGFR) and phosphorylated Akt (P-Akt) protein in esophageal squamous cell carcinoma (ESCC) were studied, and its significance in clinical prognosis of patients was assessed. The expression of P-EGFR and P-Akt protein in 83 cases of ESCC and 83 normal esophageal tissues was determined by immunohistochemical staining. Log-rank test and correlation analysis were used to analyze the prognosis of ESCC. The positive expression of P-EGFR in ESCC was $88 \%$ (73/83 cases) compared with $41 \%$ in normal esophageal mucosa $(34 / 83$ cases $)(\mathrm{P}<0.05)$. The rate of $\mathrm{P}-\mathrm{Akt}$ protein expression in ESCC was $90.4 \%$ (75/83 cases), compared with $27.7 \%$ seen in normal esophageal mucosa (23/83 cases) $(\mathrm{P}<0.05)$. The expression of $\mathrm{P}-\mathrm{EGFR}$ and $\mathrm{P}-\mathrm{Akt}$ protein was positively correlated with lymph node metastasis and degree of differentiation $(\mathrm{P}<0.05)$ irrespective of sex, age, tumor diameter and TNM stage $(\mathrm{P}>0.05)$. The expression of $\mathrm{P}-\mathrm{EGFR}$ was positively correlated with that of $\mathrm{P}-\mathrm{Akt}$ protein $(\mathrm{r}=0.674$, $\mathrm{P}<0.01)$. P-EGFR expression was negatively correlated with survival time of patients with ESCC ( $\mathrm{r}=-0.526, \mathrm{P}<0.01)$. The Kaplan-Meier survival curves showed that the cumulative survival rate of P-EGFR-positive cases was significantly lower than that of the P-EGFR-negative cases $(\mathrm{P}<0.01)$. The expression of P-Akt was negatively correlated with survival in patients with ESCC ( $\mathrm{r}=-0.473, \mathrm{P}<0.01)$. The Kaplan-Meier survival curves showed that the cumulative survival rate of the P-Akt-positive cases was significantly lower than that of the P-Akt-negative cases $(\mathrm{P}<0.01)$. In conclusion, $\mathrm{P}-\mathrm{EGFR}$
\end{abstract}

Correspondence to: Dr Qing-Xia Fan, Department of Oncology, The First Affiliated Hospital of Zhengzhou University, 1 Jianshe East Road, Zhengzhou, Henan 450052, P.R. China

E-mail: sld166399@163.com

*Contributed equally

Key words: esophageal carcinoma, phosphorylated epidermal growth factor receptor, phosphorylated Akt, immunohistochemistry, prognosis and P-Akt protein expression is closely related to the incidence of ESCC and mediates the development of invasive cancer and metastasis. It is used to determine the prognosis of ESCC, and may represent a new therapeutic target for the disease.

\section{Introduction}

Esophageal cancer is a common malignant tumor with an increasing incidence and mortality. Surgery combined with radiotherapy and chemotherapy is indicated for esophageal cancer. However, many patients are diagnosed in advanced stages of esophageal cancer, resulting in poor treatment with poor prognosis (1). Clinical practice suggests (2) that recurrence and metastasis are important factors contributing to the death of patients with esophageal cancer. Epidermal growth factor receptor (EGF) is a tyrosine kinase present on cell surface receptors, which affects several growth factors.

Phosphorylated epidermal growth factor receptor (P-EGFR) activates a variety of intracellular signaling pathways, inducing cell proliferation and survival (3). Akt signaling pathway is an important pathway regulating downstream signaling by EGFR (4). Phosphorylated Akt (p-Akt) activates Akt signaling, and the downstream genes in the signaling pathways. It controls the evolution, invasion, development and apoptosis of cancer cells.

Esophageal squamous cell carcinoma (ESCC) is a common pathological type of esophageal cancer. China has a high proportion of ESCC, prompting studies investigating the genetic mechanisms underlying the disease (5-8). However, the pathogenesis of ESCC is not very clear, and the tumor markers for the diagnosis of ESCC are few in number. Therefore, it is imperative to undertake an in-depth study into the genetic and molecular mechanisms underlying the pathogenesis of ESCC. Development of ESCC markers with strong specificity and high sensitivity, and molecular therapeutic targets has significant clinical implications. In this study, we investigated 83 cases of esophageal squamous carcinoma tissue compared with the corresponding normal esophageal mucosa, from January 2009 to October 2010 at the First Affiliated Hospital of Zhengzhou University. The expression of P-EGFR and p-Akt was detected immunohistochemically using the SP method. Its prognostic value was analyzed to provide a valuable standard of reference for diagnosis and prediction of survival. 


\section{Materials and methods}

Materials. We studied 83 cases of esophageal squamous carcinoma tissue and the corresponding normal esophageal mucosa, from January 2009 to October 2010 at the First Affiliated Hospital of Zhengzhou University. Patients included 44 males and 39 females. The age ranged from 34 to 68 years, with an average age of $56.2 \pm 33.5$ years. TNM staging revealed stage II in 23 cases, stage IIIa in 25 cases, stage IIIb in 26 cases, and stage IV in 9 cases. Poor differentiation was seen in 50 cases, moderate in 25 cases, and high in 8 cases. Lymph node metastasis was observed in 43 cases, and none in 40 cases. The tumor diameter was $<3 \mathrm{~cm}$ in 26 cases, $3-5 \mathrm{~cm}$ in 32 cases, and $>5 \mathrm{~cm}$ in 25 cases. The selected subjects were not treated with neoadjuvant therapy. Seventy patients were followed up over 60 months. This study was approved by the Ethics Committee of the First Affiliated Hospital of Zhengzhou University. Signed written informed consents were obtained from all participants before the study.

Main reagents. Rabbit monoclonal P-EGFR antibody (dilution, 1:100; cat. no. sc-120) and rabbit polyclonal p-Akt antibody (dilution: 1:100; cat. no. sc-8312) from Santa Cruz Biotechnology, Inc. (Santa Cruz, CA, USA), and xylene (Dongguan Jiabao Petroleum Chemical Co., Ltd., Dongguan, China).

Immunohistochemical assay. Sliced specimens were incubated at $58^{\circ} \mathrm{C}$ for $\sim 3 \mathrm{~h}$, and subjected to xylene treatment for dewaxing. P-EGFR was detected as follows using microwave repair at $650 \mathrm{~W}$ for $20 \mathrm{~min}$ and cooled to room temperature, washed 3 times using Tween-phosphate-buffered saline (PBS), first at $25^{\circ} \mathrm{C}$ for $16 \mathrm{~h}$, and washed three times by PBS, followed by $\mathrm{DAB}$ for coloration, and counterstained with hematoxylin. After dehydration, it was observed under a light microscope (BX-42; Olympus, Tokyo, Japan). PBS was used as a substitute for the first antibody and as the negative control for P-EGFR detection. The positive film was confirmed by Santa Cruz Biotechnology, Inc. that provided the rabbit anti-human P-EGFR as a positive control group.

The p-Akt was detected using boiling repair at $98^{\circ} \mathrm{C}$ for $20 \mathrm{~min}$, cooled to room temperature, and washed 3 times with PBS. After incubation with the first antibody at $4{ }^{\circ} \mathrm{C}$ for $16 \mathrm{~h}$, it was washed three times with PBS, and DAB for coloration, followed by hematoxylin for counterstaining. After dehydration the specimen was visualized under a light microscope. PBS was used as a substitute for the first antibody with P-EGFR as the negative control. The positive film was confirmed by Santa Cruz Biotechnology, Inc. that provided rabbit anti-human p-Akt as a positive control group.

Evaluation criterion. In the cytoplasm, cell membrane and nucleus, the yellow particles were found to be positive cells. Each slice was randomly selected under 5 high power fields, and the number of positive cells in 100 cells was calculated.

Scoring criteria for P-EGFR expression level (9): Absence of staining or $<5 \%$ tumor cell staining was scored as (-); 5-19\% of tumor cell staining was considered as $(+) ; 20-50 \%$ was scored as $(++)$, and $>50 \%$ tumor cell staining was determined as $(+++)$.

Scoring criteria for p-Akt expression were (10): No staining or $<30 \%$ staining was determined as $(-),>30 \%$ tumor cell staining
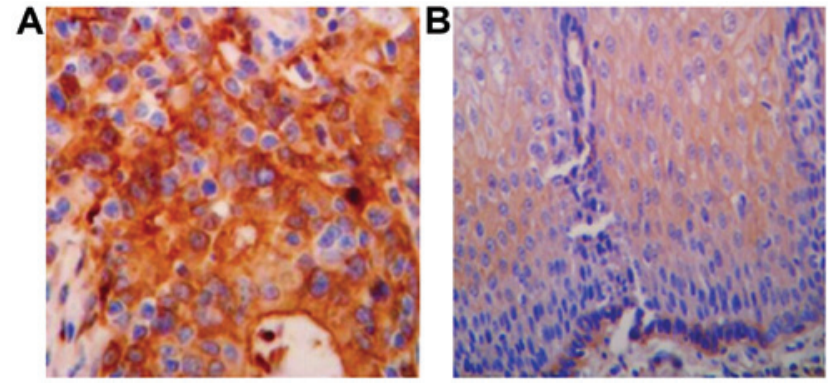

Figure 1. Expression of P-EGFR protein (SP, x400). (A) ESCC; (B) adjacent normal esophageal tissue. P-EGFR, phosphorylated epidermal growth factor receptor; ESCC, esophageal squamous cell carcinoma tissue.

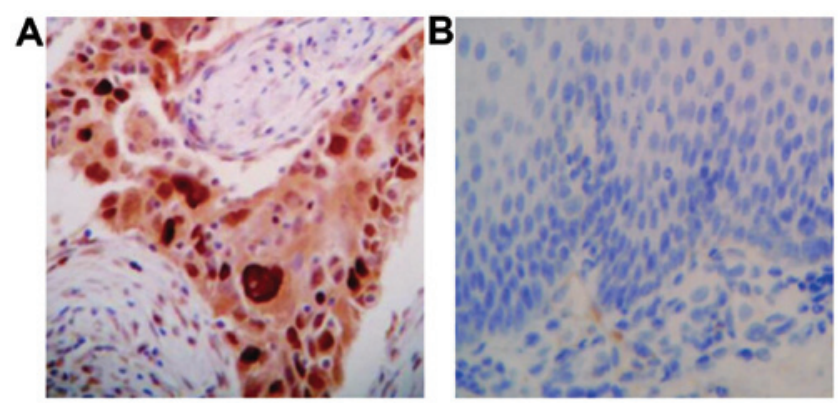

Figure 2. Expression of p-Akt protein (SP, x400). (A) ESCC tissue; (B) adjacent normal esophageal tissue. ESCC, esophageal squamous cell carcinoma tissue.

was determined as positive, yellow as $(+)$, brownish yellow as $(++)$, and brown as $(+++)$. The final results were as follows: $(-)$ denoted negative expression (PE-GFR, p-Akt); and $(+),(++)$ and $(+++)$ represented positive expression $\left(\mathrm{PE}-\mathrm{GFR}^{+}, \mathrm{p}-\mathrm{Akt}^{+}\right)$.

Statistical analysis. The experimental data were analyzed by SPSS 21.0 software (IBM, Armonk, NY, USA). The two factors were analyzed by non-parametric Spearman's rank correlation. Kaplan-Meier method was used to analyze the survival based on single factor, and the difference was tested by log-rank test. $\mathrm{P}<0.05$ indicated statistical significance. The survival curve was used for analysis of the follow-up time and the survival condition of the patients.

\section{Results}

Expression and distribution of P-EGFR and P-Akt protein. P-EGFR was mainly expressed in the cytoplasm or cell membrane, with the positive expression was represented by brown yellow particles (Fig. 1). The P-Akt protein was expressed in the cytoplasm or nucleus, and the positive expression was visualized as brownish yellow particles (Fig. 2).

The rate of positive expression of P-EGFR in ESCC was $88 \%$ (73/83 cases), which was significantly higher than the rate of $41 \%$ in the normal esophageal mucosa $(34 / 83$ cases $)(\mathrm{P}<0.05)$ (Table I).

The rate of P-Akt protein expression in ESCC was $90.4 \%$ (75/83 cases), which was significantly higher than in the normal esophageal mucosa $(27.7 \%$; $23 / 83$ cases $)(\mathrm{P}<0.05)$ (Table II).

Expression of $P$-EGFR and P-Akt protein and clinical pathology of patients with ESCC. The expression of P-EGFR 
Table I. Expression of P-EGFR in esophageal squamous cell carcinoma and adjacent normal esophageal tissues n (\%).

\begin{tabular}{lccccc}
\hline Tissue & $\mathrm{n}$ & + & ++ & +++ & Total \\
\hline ESCC tissue & 83 & $16(19.3)$ & $20(24.1)$ & $37(44.6)$ & $73(88)$ \\
Adjacent normal esophageal tissue & 83 & $24(28.9)$ & $6(7.2)$ & $4(4.8)$ & $34(41)$ \\
$\chi^{2}$ & & & & $49(59)$ \\
P-value & & & & 16.753 \\
\hline
\end{tabular}

P-EGFR, phosphorylated epidermal growth factor receptor; ESCC, esophageal squamous cell carcinoma.

Table II. Expression of p-Akt in ESCC and adjacent normal esophageal tissues n (\%).

\begin{tabular}{|c|c|c|c|c|c|c|}
\hline Tissue & $\mathrm{n}$ & + & ++ & +++ & Total & - \\
\hline ESCC tissue & 83 & $18(21.7)$ & $20(24.1)$ & $37(44.6)$ & $75(90.4)$ & $8(9.6)$ \\
\hline Adjacent normal esophageal tissue & 83 & $17(20.5)$ & $4(4.8)$ & $2(2.4)$ & $23(27.7)$ & $60(72.3)$ \\
\hline$\chi^{2}$ & & & & & & 33.625 \\
\hline P-value & & & & & & $<0.05$ \\
\hline
\end{tabular}

P-Akt, phosphorylated Akt; ESCC, esophageal squamous cell carcinoma.

Table III. Pathology of esophageal squamous cell carcinoma: Expression of P-EGFR and P-Akt.

\begin{tabular}{|c|c|c|c|c|c|}
\hline $\begin{array}{l}\text { Clinical pathological } \\
\text { characteristics }\end{array}$ & $\mathrm{n}$ & P-EGFR-positive cases & $\chi^{2}(\mathrm{P}$-value $)$ & P-Akt-positive cases & $\chi^{2}(\mathrm{P}$-value $)$ \\
\hline \multicolumn{6}{|l|}{ Sex } \\
\hline Male & 44 & 38 & $0.342(>0.05)$ & 39 & $0.351(>0.05)$ \\
\hline Female & 39 & 35 & & 36 & \\
\hline \multicolumn{6}{|l|}{ Tumor diameter, $\mathrm{cm}$} \\
\hline$<3$ & 26 & 23 & $1.163(>0.05)$ & 23 & $2.505(>0.05)$ \\
\hline $3-5$ & 32 & 28 & & 29 & \\
\hline$>5$ & 25 & 22 & & 23 & \\
\hline \multicolumn{6}{|l|}{ TNM staging } \\
\hline II stage & 23 & 21 & $5.326(>0.05)$ & 21 & $1.634(>0.05)$ \\
\hline IIIa stage & 25 & 22 & & 23 & \\
\hline IIIb stage & 26 & 23 & & 24 & \\
\hline IV stage & 9 & 7 & & & \\
\hline \multicolumn{6}{|c|}{ Degree of differentiation } \\
\hline Low & 50 & 50 & $9.741(<0.05)$ & 49 & $9.266(>0.05)$ \\
\hline Middle & 25 & 19 & & 22 & \\
\hline High & 8 & 4 & & 4 & \\
\hline \multicolumn{6}{|l|}{ Lymph node metastasis } \\
\hline Yes & 43 & 42 & $8.176(<0.05)$ & 43 & $6.282(<0.05)$ \\
\hline No & 40 & 31 & & 32 & \\
\hline
\end{tabular}

P-EGFR, phosphorylated epidermal growth factor receptor; P-Akt, phosphorylated Akt.

and P-Akt protein in patients with ESCC was correlated with lymph node metastasis and degree of differentiation $(\mathrm{P}<0.05)$, irrespective of sex, age, tumor diameter or TNM stage $(\mathrm{P}>0.05$; Table III).
Expression of P-EGFR and P-Akt protein in ESCC. Pearson correlation analysis showed that the expression of P-EGFR was positively correlated with that of P-Akt protein in ESCC $(\mathrm{r}=0.674, \mathrm{P}<0.01)$ (Fig. 3). 


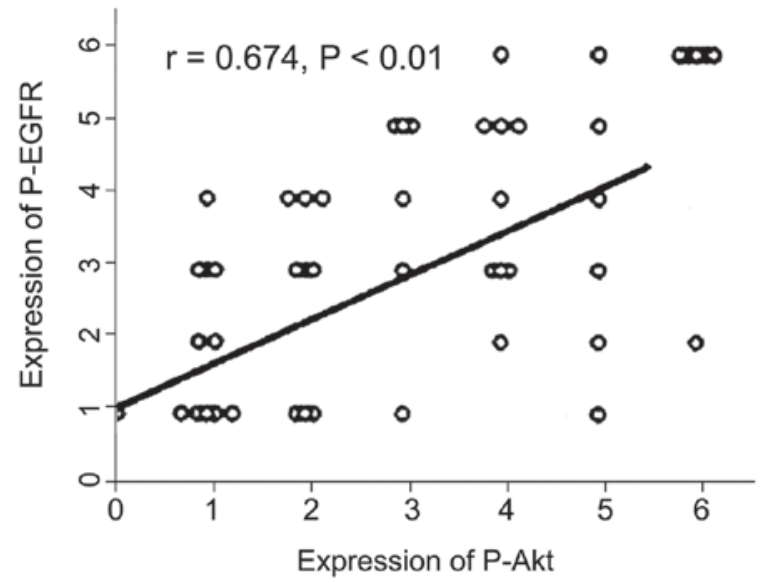

Figure 3. Correlation of P-EGFR and P-Akt expression in ESCC. P-EGFR, phosphorylated epidermal growth factor receptor; ESCC, esophageal squamous cell carcinoma tissue.

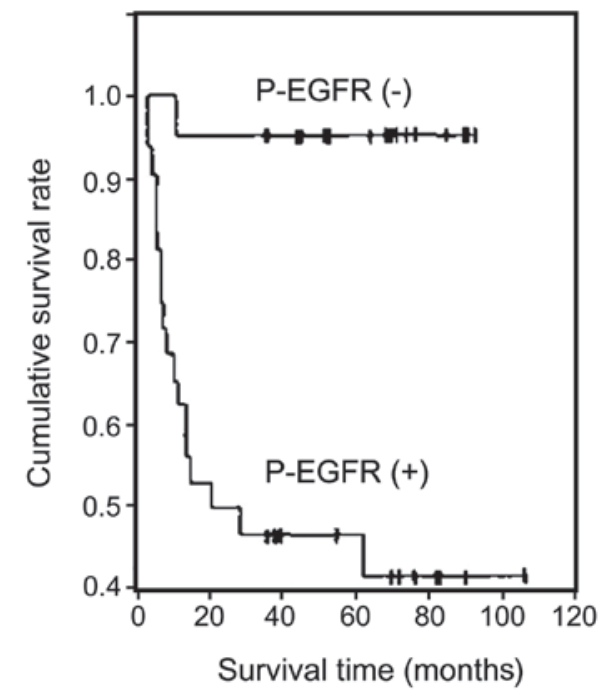

Figure 4. Survival curve of P-EGFR and prognosis of ESCC. P-EGFR, phosphorylated epidermal growth factor receptor; ESCC, esophageal squamous cell carcinoma tissue.

Relationship between P-EGFR and P-Akt expression with survival time in patients with ESCC. P-EGFR expression was negatively correlated with survival time in patients with ESCC $(r=-0.526, P<0.01)$. Kaplan-Meier survival curves showed that the cumulative survival rate of the P-EGFR-positive cases was significantly lower than that of the P-EGFR-negative cases $(\mathrm{P}<0.01)$ (Fig. 4).

The expression of P-Akt was negatively correlated with survival in patients with ESCC $(r=-0.473, \mathrm{P}<0.01)$. Kaplan-Meier survival curves showed that the cumulative survival rate of P-Akt-positive cases was significantly lower than that of the P-Akt-negative cases $(\mathrm{P}<0.01)$ (Fig. 5).

\section{Discussion}

Esophageal cancer is a common malignant tumor of the digestive tract, with a poor prognosis. It is generally divided into squamous cell carcinoma and adenocarcinoma (11). However, there are regional differences in the distribution of

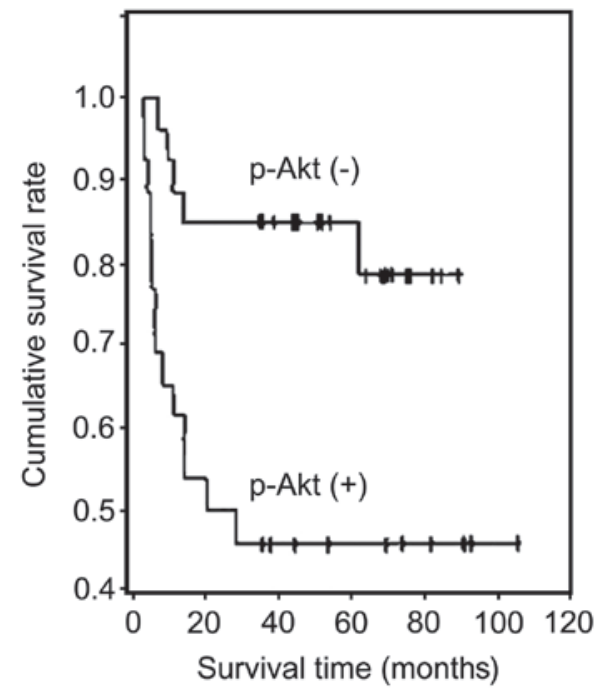

Figure 5. Survival curve of P-Akt and prognosis of ESCC. ESCC, esophageal squamous cell carcinoma tissue.

esophageal cancer: Adenocarcinoma is prevalent in Europe and America, but squamous cell carcinoma occurs predominantly in China. In recent years, several studies have investigated esophageal cancer (12-14). Clinical studies show (15) that patients with ESCC are treated with chemotherapy. Results show that the esophageal cancer is triggered by multiple factors in multiple stages. The developmental course is divided into simple hyperplasia, atypical hyperplasia, carcinoma in situ and infiltrative cancer. A series of oncogenes and anti-oncogenes are expressed. N-methyl-N nitrosourea alkyl induces ESCC in rats. Experimental studies confirmed the occurrence of esophageal cancer following long-term exposure to specific carcinogens (16). Therefore, researchers proposed several models, such as nitrosamine carcinogenic model, 4-nitroquinoline-oxide model, ectopic transplantation, ESCC model, and orthotopic transplantation of ESCC. Studies investigated the etiology and development of ESCC. However, the specific regulatory mechanism of ESCC and its pathogenesis remain obscure. The absence of effective clinical treatment resulted in a high incidence of ESCC, poor clinical prognosis, and high mortality rate.

Advances in molecular biology have shed new light on the molecular markers of prognosis in ESCC, including the expression of Fn14, VEGF, NGX6, COX-2, cyclin D1, E-cadherin, and IMP3. The value of prognosis in ESCC is established. Recent studies have indicated that $(1,17)$ a high expression of EGFR is related to prognosis of nasopharyngeal carcinoma. Therefore, we investigated the molecular targeted therapies of cancer. Using EGFR as molecular targets, drugs such as erlotinib and cetuximab have been developed. P-EGFR belongs to the active form of EGFR. Studies have reported that EGFR itself is not an important factor in cancer (such as nasopharyngeal) cell proliferation. Elevated P-EGFR expression plays a key role in the prevalence of cancer, and induces the proliferation of cancer cells. However, researchers investigating gastrointestinal carcinoid and pancreatic cancers detected increased expression of P-EGFR and EGFR proteins. The study also found that pancreatic cancer patients with low or no expression of P-EGFR showed better prognosis than 
patients with high expression of P-EGFR (18). Akt is highly activated in tumors suggesting that the growth, differentiation and proliferation of tumor cells, was abnormal. In vitro studies suggest that the phosphorylation of Akt residues threonine 308 and serine 473 was closely related to the activation of PI3K/Akt signaling (19). However, the role and clinical significance of P-Akt in the occurrence, development and evolution of tumors in the human body is not very clear.

Cancer specimens derived from pathological archives of immunohistochemical staining revealed gene products in patients with tumor, and retrospective analysis of clinical data is an important approach of clinical investigation. Tumor dissemination in the body caused by cancer metastasis is refractory to surgical treatment. Therefore, it is imperative to understand the factors associated with tumor metastasis, and understand the mechanisms underlying invasive cancer, to predict cancer metastasis and clinical treatment.

In this study, we analyzed P-EGFR and P-Akt expression in ESCC tissues and in the corresponding normal esophageal mucosa immunohistochemically. We found a P-EGFR positive expression rate of $88 \%$ in cancer tissues of ESCC, which was significantly higher than the $41 \%$ found in normal esophageal mucosa tissues $(\mathrm{P}<0.05)$. The positive rate of $\mathrm{P}-\mathrm{Akt}$ protein expression in the cancer tissue of patients with ESCC was $90.4 \%$, which was significantly higher than in the corresponding normal esophageal mucosa tissues, at $27.7 \%$ $(\mathrm{P}<0.05)$. The positive rate of $\mathrm{P}-\mathrm{Akt}$ and $\mathrm{P}-\mathrm{EGFR}$ protein expression in ESCC is correlated with lymph node metastasis and differentiation $(\mathrm{P}<0.05)$ independent of sex, age, tumor diameter and TNM stage $(\mathrm{P}>0.05)$. The level of P-Akt and P-EGFR expression may be closely correlated with the occurrence and evolution of ESCC.

Our analysis showed that the P-EGFR and P-Akt protein expression in ESCC was positively correlated $(r=0.674$, $\mathrm{P}<0.01)$. P-EGFR and P-Akt show a synergistic effect in regulating the proliferation and survival of ESCC cells in vivo.

We analyzed the follow-up data and survival time. The results suggest that the expression of P-EGFR was negatively correlated with the survival time of patients with ESCC $(r=-0.526, P<0.01)$. Kaplan-Meier survival curves showed that the cumulative survival rate of the P-EGFR-positive cases was significantly lower than that of the P-EGFR-negative cases $(\mathrm{P}<0.01)$. The expression of $\mathrm{P}-\mathrm{Akt}$ was negatively correlated with survival in patients with $\operatorname{ESCC}(\mathrm{r}=-0.473, \mathrm{P}<0.01)$. Kaplan-Meier survival curves showed that the cumulative survival rate of P-Akt-positive cases was significantly lower than that of the P-Akt-negative cases $(\mathrm{P}<0.01)$. P-Akt and P-EGFR promoted metastasis of ESCC and shortened the survival of patients with ESCC.

In conclusion, the high expression of P-Akt and P-EGFR is related to lymph node metastasis and differentiation of ESCC. P-Akt and P-EGFR represent markers of ESCC. The combination of P-EGFR and P-Akt levels is helpful in evaluating the severity of ESCC and predicting their survival time.

\section{References}

1. Aleskandarany MA, Rakha EA, Ahmed MA, Powe DG, Ellis IO and Green AR: Clinicopathologic and molecular significance of phospho-Akt expression in early invasive breast cancer. Breast Cancer Res Treat 127: 407-416, 2011.
2. Hembrough T, Thyparambil S, Liao WL, Darfler MM, Abdo J, Bengali KM, Taylor P, Tong J, Lara-Guerra H, Waddell TK, et al: Selected reaction monitoring (SRM) analysis of epidermal growth factor receptor (EGFR) in formalin fixed tumor tissue. Clin Proteomics 9: 5, 2012.

3. Abdulwahab A, Sykes J, Kamel-Reid S, Chang H and Brandwein JM: Therapy-related acute lymphoblastic leukemia is more frequent than previously recognized and has a poor prognosis. Cancer 118: 3962-3967, 2012

4. Licitra L, Störkel S, Kerr KM, Van Cutsem E, Pirker R, Hirsch FR, Vermorken JB, von Heydebreck A, Esser R, Celik I, et al: Predictive value of epidermal growth factor receptor expression for first-line chemotherapy plus cetuximab in patients with head and neck and colorectal cancer: analysis of data from the EXTREME and CRYSTAL studies. Eur J Cancer 49: 1161-1168, 2013.

5. Jia M and Souchelnytstkyi S: Comments on the cross-talk of TGF $\beta$ and EGF in cancer. Exp Oncol 33: 170-173, 2011.

6. Mints M and Souchelnytskyi S: Impact of combinations of EGF, TGF $\beta, 17 \beta$-oestradiol, and inhibitors of corresponding pathways on proliferation of breast cancer cell lines. Exp Oncol 36: 67-71, 2014

7. Cree IA: Designing personalised cancer treatments. J Control Release 172: 405-409, 2013.

8. Moghadamtousi SZ, Kadir HA, Paydar M, Rouhollahi E and Karimian H: Annona muricata leaves induced apoptosis in A549 cells through mitochondrial-mediated pathway and involvement of NF-кB. BMC Complement Altern Med 14: 299, 2014.

9. Asare GA, Afriyie D, Ngala RA, Abutiate H, Doku D, Mahmood SA and Rahman H: Antiproliferative activity of aqueous leaf extract of Annona muricata L. on the prostate, BPH-1 cells, and some target genes. Integr Cancer Ther 14: 65-74, 2015.

10. Mimeault M and Batra SK: Frequent gene products and molecular pathways altered in prostate cancer- and metastasis-initiating cells and their progenies and novel promising multitargeted therapies. Mol Med 17: 949-964, 2011.

11. Mimeault M, Johansson SL and Batra SK: Pathobiological implications of the expression of EGFR, pAkt, NF- $\kappa$ B and MIC-1 in prostate cancer stem cells and their progenies. PLoS One 7: e31919, 2012.

12. Llovet P, Sastre J, Ortega JS, Bando I, Ferrer M, García-Alfonso P, Donnay O, Carrato A, Jiménez A, Aranda E, et al: Prognostic value of BRAF, PI3K, PTEN, EGFR copy number, amphiregulin and epiregulin status in patients with KRAS codon 12 wild-type metastatic colorectal cancer receiving first-line chemotherapy with anti-EGFR therapy. Mol Diagn Ther 19: 397-408, 2015.

13. Pentheroudakis G, Kotoula V, De Roock W, Kouvatseas G, Papakostas P, Makatsoris T, Papamichael D, Xanthakis I, Sgouros J, Televantou D, et al: Biomarkers of benefit from cetuximab-based therapy in metastatic colorectal cancer: interaction of EGFR ligand expression with RAS/RAF, PIK3CA genotypes. BMC Cancer 13: 49, 2013.

14. Loupakis F, Cremolini C, Fioravanti A, Orlandi P, Salvatore L, Masi G, Schirripa M, Di Desidero T, Antoniotti C, Canu B, et al: EGFR ligands as pharmacodynamic biomarkers in metastatic colorectal cancer patients treated with cetuximab and irinotecan. Target Oncol 9: 205-214, 2014.

15. Petrelli F, Borgonovo K and Barni S: The predictive role of skin rash with cetuximab and panitumumab in colorectal cancer patients: a systematic review and meta-analysis of published trials. Target Oncol 8: 173-181, 2013.

16. Li P, Yang R and Gao WQ: Contributions of epithelial-mesenchymal transition and cancer stem cells to the development of castration resistance of prostate cancer. Mol Cancer 13: 55, 2014.

17. Pan Y, Zhang Y, Li Y, Hu H, Wang L, Li H, Wang R, Ye T, Luo X, Zhang Y, et al: Prevalence, clinicopathologic characteristics, and molecular associations of EGFR exon 20 insertion mutations in East Asian patients with lung adenocarcinoma. Ann Surg Oncol 21 (Suppl 4): S490-S496, 2014.

18. Cheng L, Ren W, Xie L, Li M, Liu J, Hu J, Liu BR and Qian XP: Anti-EGFR MoAb treatment in colorectal cancer: limitations, controversies, and contradictories. Cancer Chemother Pharmacol 74: 1-13, 2014.

19. Nishikawa M, Miyake H and Masato F: Enhanced sensitivity to sunitinib by inhibition of Akt-1 expression in human castration-resistant prostate cancer PC 3 cells both in vitro and in vivo. Mol Cancer Ther 13: 1949-1960, 2014. 